\title{
Improved Resuscitation Method for Choking Victims
}

\section{Puthalath Koroth Raghuprasad}

Independent Researcher, Odessa, USA

Email: pkrp12@gmail.com

How to cite this paper: Raghuprasad, P.K (2021) Improved Resuscitation Method for Choking Victims. Open Journal of Emergency Medicine, 9, 143-149. https://doi.org/10.4236/ojem.2021.94014

Received: September 11, 2021

Accepted: October 16, 2021

Published: October 19, 2021

Copyright $\odot 2021$ by author(s) and Scientific Research Publishing Inc. This work is licensed under the Creative Commons Attribution International License (CC BY 4.0).

http://creativecommons.org/licenses/by/4.0/

\begin{abstract}
Abdominal thrust, popularized by Henry Heimlich in 1975 is the standard resuscitation method for victims of choking. When properly applied, this procedure can and has saved many lives over the years, but the process itself is somewhat difficult to perform. The main reason for this difficulty is the need to reach around the trunk of the victim and then apply sufficient force to generate enough pressure upwards to the diaphragm, to be able to dislodge the food bolus (or foreign body) out of the larynx. This is compounded by the size of the average American, especially when they are overweight or obese, thus the ability to reach the front of the abdomen and then the prospect of actually trying to lift the victim up through brute force is a challenge for most people. Some difficulties are inherent in the emotionally charged and panicky situations in which such resuscitation measures take place. Some scientists have advocated the use of mechanical devices to apply thrust to the abdomen. However, the lack of availability of such assisting devices at home and even in restaurants is a distinct issue. Damage to the xiphisternum or internal organs by excessive force is also an issue, so much so, most authorities recommend a post-resuscitation check up of all victims at a hospital or clinic. The procedure(s) described in this report is much simpler in execution, while being almost effortless, and applicable to victims of any size. It also exploits the objects that are likely to be present where such choking accidents occur, the restaurants and dining rooms, while also taking advantage of the earth's gravity to dislodge the offending food bolus. If such objects are not available, an alternate method using the rescuer's free forearm to support the victim's weight, while keeping him/her folded over is also described.
\end{abstract}

\section{Keywords}

Abdominal Thrust, Choking, Food Bolus, Heimlich Maneuver, Resuscitation 


\section{Introduction}

The method of the abdominal thrusts introduced by Dr. Heimlich is for an operator to stand behind the victim, wrap the arms around the waist and place a closed fist in the space between the umbilicus and the sternum, then use the other hand to clasp the closed fist and exert abrupt pressure upward and towards the operator, as if attempting to lift the victim up [1]. Emergency Medical Services must be called as soon as possible, but without delaying attempts at resuscitation. While the use of Heimlich maneuver is still the standard procedure on adults who are conscious but unable to expel the food bolus by coughing voluntarily, some modifications have been recommended by some authorities [2] [3] [4]. The American Red Cross [5], Mayo Clinic [6] and The UK National Health Service (NHS) [7] suggest applying 5 slaps on the back of the victim after bending them forward. If these fail to expel the obstructing foreign body, repeat the processes until either is successful or the Emergency Medical Staff arrive. The American Heart Association and the American Red Cross (recently) omitted the back slaps; the Australian and New Zealand Resuscitation Council have omitted the abdominal thrusts, for fear of complications, but have continued to recommend the back slaps. When the choking victim is alone, as back slaps are impossible, "auto abdominal thrusts" have been advocated; these are similar to the usual abdominal thrusts but the victims themselves administer them.

Attempts to improve or modify the resuscitation methods have involved, in obese and pregnant victims, to apply the thrusts around the mid-chest region [6] or to around the base of the sternum [8]; for infants the recommendation is to straddle them prone on the performer's forearm and do the abdominal thrusts in that recumbent position [8] after 5 back blows are done gently with the heel of the hand. Another recommendation is to use the top of the back of a chair or even against a countertop, at the same location as in abdominal thrusts, to apply abrupt pressure onto the abdomen. Several "choking-assistance devices" have also been invented [9] [10] [11].

A group from the United Kingdom reported the results of a study in thorax.bmj.com [12] on the intra-abdominal pressure induced by various measures. These measures included circumferential/horizontal abdominal thrusts, Heimlich maneuver that incorporates the upward and inward thrusts, "Auto-up-thrusts", abdominal thrusts, "chair thrusts" as described above, and "volitional maximal cough and sniff pressure". The most increase in the intra-abdominal pressure was recorded with the "chair thrusts" with the help of a "rescuer", as well as when such thrusts were done by the victim themselves.

In this paper, the current author incorporates the best features of the reported methods, as far as effectiveness, paying attention to also the ease of performance and the need for the least physical force. Also, and most importantly, by taking advantage of the effect of gravity, this technique is potentially more effective, is least damaging, and available to all victims in most circumstances where choking takes place over a chunk of food or another foreign body. Further, there is no 
requirement of any special devices, as chairs and countertops are usually present for performing the "thrust", at places where food is consumed, such as one's dining room or at restaurants. A bonus of this technique is the reduced likelihood of injuries to sternum or any internal organs.

\section{Materials and Methods}

The only material(s) required is a chair or sofa with a sturdy back, or a countertop. If a rescuer is available, they can help by both contacting emergency rescue team, administering the "thumps" after positioning the victim in the appropriate position and in accomplishing the required thrust against the top of the back rest or on a countertop or, in case a chair is not available, by administering the thrust against the rescuer's own forearm (as shown in Figure 4(b)).

The methods of administering the resuscitation can be described under 3 broad classes. In all the accompanying figures and descriptions a chair is used but any of the other agents described above can be used instead; thus, when the text mentions "chair", it could be one of the other props mentioned in this paper elsewhere.

1) When a rescuer is available. After calling the emergency professionals, have the victim lean over the back of the chair in an inverted " $U$ " position, such that the whole of the thorax and head are essentially upside down (See Figure 1 \& Figure 2).

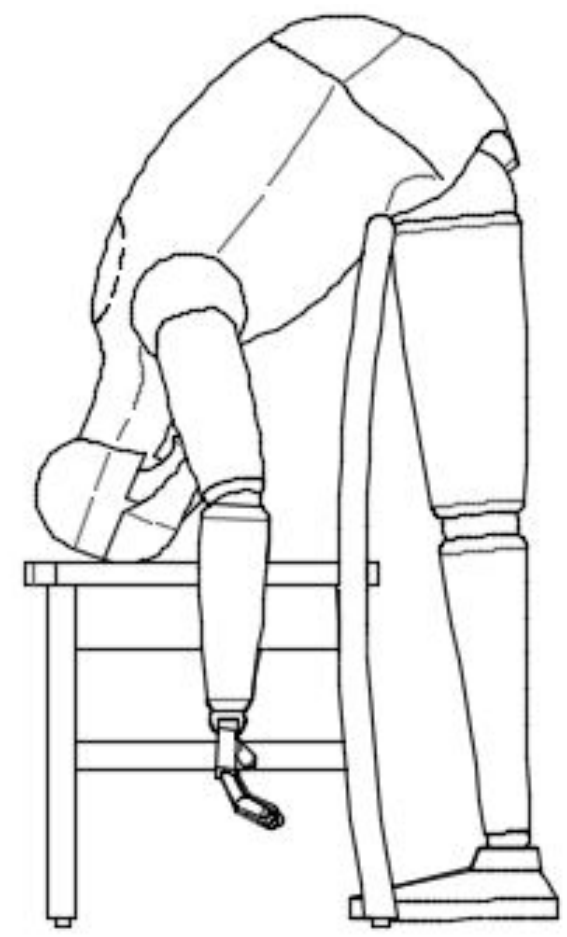

Figure 1. This is a lateral view of the position of the victim showing the inverted " $U$ " achieved by bending over the top of the backrest of the chair and allowing the victim's head to rest on the chair. Partial view of the oval with interrupted lines below the root of the neck is just visible; this is the location for administering the thumps. 


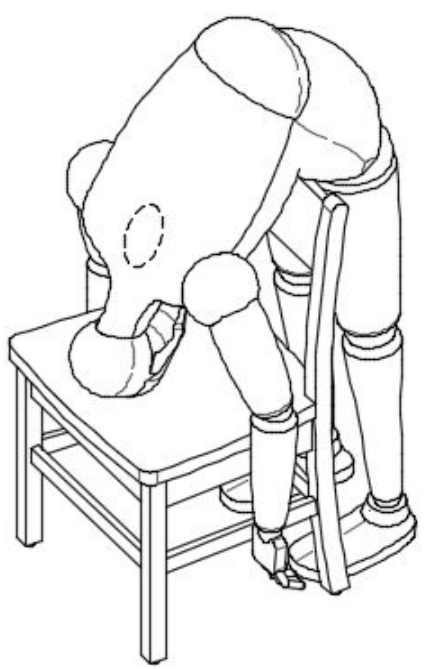

Figure 2. Position of the victim shown as viewed from the front and to the left; the oval area with interrupted lines just below the root of the neck is where the thumps are to be administered.

This accomplishes two objectives. First, the upside down position of the upper torso takes advantage of gravity to begin dislodging the food bolus or foreign body from the vocal cords, which represent the narrowest portion of the larynx where they are lodged. Second, the weight of the victim presses on the top of the backrest, to apply the "thrust". Next, as shown in Figure 3, the rescuer administers abrupt thumps with a closed fist at the location marked by the interrupted oval just inferior to the root of the neck. It is anticipated that, after one or two blows, the obstructing material will be dislodged and expelled.

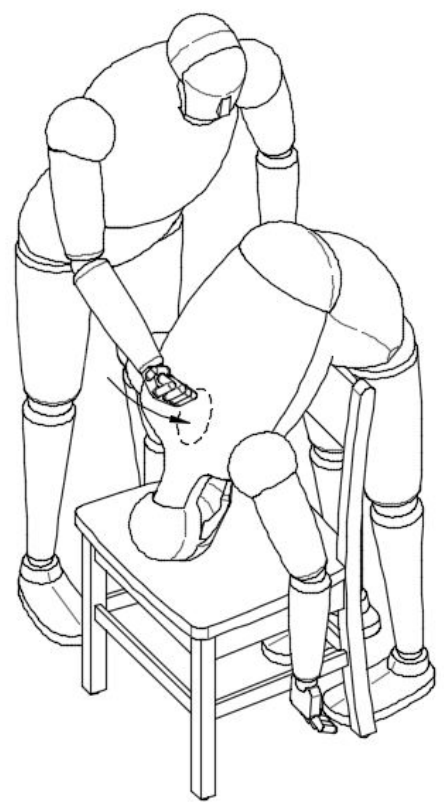

Figure 3. Shown in this figure is the method of administering the "thumps" at below the root of the neck, with the victim positioned in the inverted " $U$ ", with their own weight pressing against the top of the backrest to automatically apply the "thrust". 
2) When the choking victim is alone. Figure $1 \&$ Figure 2 still apply. The victim places themselves over the chair and intentionally presses down at the backrest, pressing down repeatedly. Of course, impossible is the thumping on the back.

3) When no chair is available. Figure 4(a) and Figure 4(b) show two versions of the method.

The preferred version is in Figure 4(b), as the rescuer can both support the weight of the victim and at the same time apply upward thrusting motions onto the abdomen.

The same methods as above can be used for obese persons, babies and on victims who have become unconscious. In pregnant women, the preferred method might be as shown in Figure 4(b) (rescuer without chair back rest), with the supporting arm stretched across the lower part of the thorax. A suitable adaptation in babies might be holding the victim upside down, holding by both ankles and administering gentle thumps around the back of the root of the neck.

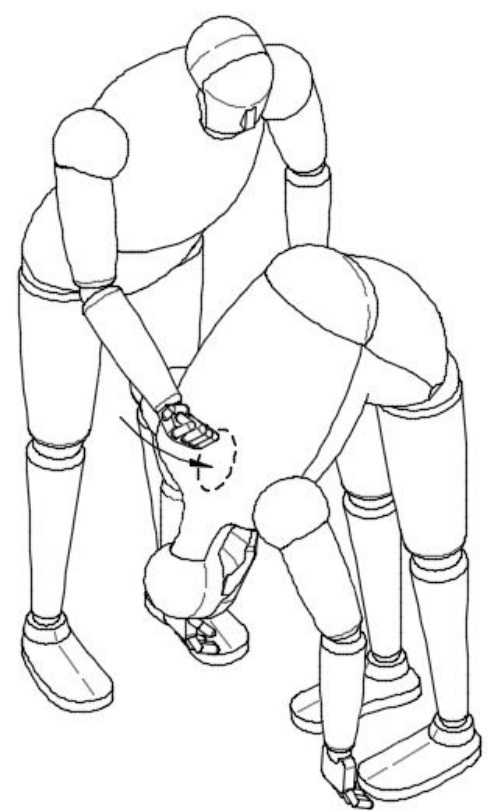

(a)

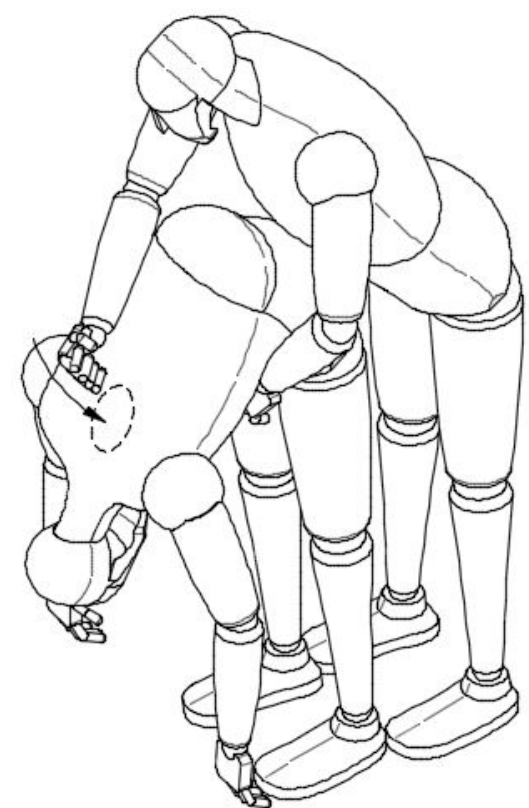

(b)

Figure 4. (a) \& (b) show slightly different ways to administer the thumps by a rescuer, when no "chair" is available. It is anticipated that (b) represents the preferred way, as one arm of the rescuer can both support the victim, and apply the thrust against the abdomen, while the other fist is used to administer the thumps.

\section{Discussion}

As choking accidents occur without warning and, if appropriate action is not taken quickly, within a span of $4-6$ minutes, the victims can die or become permanently brain damaged, any resuscitation measure should be applied quickly. Equally important is the need for laypersons everywhere to become cognizant and comfortable in the process involved in resuscitating victims. This 
necessarily also means that the technique should be simple to execute and immediately available, without the need for any cumbersome equipment. The procedures reported in this article were developed with these requirements in mind, and we feel comfortable in stating that all our objectives are adequately met by these procedures. Because of the simplicity of this method(s), we expect most laypersons to become comfortable with it within a short period of training, perhaps as short as 30 minutes. Discussed below are some of the special features of our resuscitation techniques that make them easy to execute and efficient, and contrast them with the existing methods.

The main objection to the Heimlich procedure, that of the difficulty to efficiently wrap the rescuer's arms around obese victims, or the need to attempt to lift upward such victims, is eliminated. The objections of some authorities on advocating the "back-slaps" as potentially risky of making the foreign body to lodge deeper into the larynx or even into the trachea, is avoided by the victims' inverted position over the chair backrest. Next, the ready availability of a chair, sofa or a countertop against which the "abdominal thrusts" can be administered, is also a definite advantage. Taking advantage of earth's gravity, at the same time as applying the abdominal thrusts simply by exploiting the weight of the victims themselves, makes this technique efficient.

In order for these techniques to succeed and prevent all deaths from choking, even laypersons should learn and be comfortable in applying them to the victims. In addition to teaching these to rescuers, these simple techniques should be taught in schools and colleges and to all members of staff of restaurants and of the hospitality industry in general. Thus, hopefully in the foreseeable future, no one dies from the not-so-rare but deadly events of choking, by adopting our resuscitation methods.

\section{Conclusion}

Ideally, any method of resuscitation of choking victims should be simple and easy to administer, effective in dislodging the offending foreign body from the larynx, not require special equipment that would be unavailable at the usual locations where such emergencies arise, and most of the general public should be cognizant of the procedure(s). The resuscitation method described in this paper fulfills all these goals and we hope, will exceed them. Further, we hope that these resuscitation efforts are convenient and efficient on even the babies and pregnant women. Since some conventional resuscitation methods leave victims with injuries, the standard of care is to send all the victims to a hospital after the resuscitation methods to rule out such injuries. That may not be necessary after the resuscitation efforts described in this paper, thus saving the added expense and the needless delay in returning to regular activities.

\section{Acknowledgements}

I wish to acknowledge the excellent secretarial assistance of Ms. Rosie Luna, and 
Mr. Jeff Nichols at ididraft01@gmail.com for the five figures presented in this manuscript.

\section{Conflicts of Interest}

The author declares no conflicts of interest regarding the publication of this paper.

\section{References}

[1] Heimlich, H.J., Hoffman, KA. and Canestri, F.R. (1975) Food-Choking and Drowning Deaths Prevented by External Sub-Diaphragmatic Compression: Physiological Basis. The Annals of Thoracic Surgery, 20, 188-195. https://doi.org/10.1016/S0003-4975(10)63874-X

[2] Perkins, G.D., Handley, A.J., Koster, R.W., et al. (2015) European Resuscitation Council Guidelines for Resuscitation, 2015: Section 2. Adult Basic Life Support and Automated External Defibrillation. Resuscitation, 95, 81-99.

[3] Australian and New Zealand Resuscitation Councils (2016) ANZOR Guideline 4-Airway. https://resus.org.au/guidelines

[4] Lippman, J., Taylor, D.M., Slocombe, R., et al. (2013) Lateral versus Anterior Thoracic Thrusts in the Generation of Airway Pressure in Anaesthetized Pigs. Resuscitation, 84, 515-519. https://doi.org/10.1016/j.resuscitation.2012.11.024

[5] https://www.nhs.uk/common-health-questions/accidents-first-aid-and-treatments/ what-should-i-do-if-someone-is-choking/

[6] STEP 3: Be Informed-Conscious Choking I Be Red Cross Ready. http://www.redcross.org/flash/brr/English-html/conscious-choking.asp

[7] Mayo Clinic Staff (2011) Foreign Object Inhaled: First Aid. http://www.mayoclinic.com/health/first-aid/FA00064

[8] https://www.healthline.com/health/heimlich-maneuver

[9] Urso, C.L. (2020) Self-Rescue Anti-Choking Implements. US Patent No. 10,524,979 B1.

[10] Karpel, L.M. (2004) Choking Assistance Device. US Patent No. 6,725,863 B2.

[11] Ash, P.M. (1980) Object Dislodging Method and Apparatus. US Patent No. 4,182,317.

[12] Pavitt, M.J., Swanton, L.L., Hind, M., Apps, M., et al. (2017) Choking on a Foreign Body: A Physiological Study of the Effectiveness of Abdominal Thrust Manoeuvres to Increase Thoracic Pressure. Thorax, 72, 576-578.

https://thorax.bmj.com/contest/72/6/576 https://doi.org/10.1136/thoraxjnl-2016-209540 